\title{
RESEARCH
}

Open Access

\section{Mesenchymal stem cell-derived exosomes improve motor function and attenuate neuropathology in a mouse model of Machado-Joseph disease}

Hua-Jing You ${ }^{1 \dagger}$, Shu-Bin Fang ${ }^{2 \dagger}$, Teng-Teng Wu' ${ }^{1}$, Hongyu Zhang ${ }^{2}$, Yu-Kun Feng ${ }^{3}$, Xue-Jiao Li ${ }^{4}$, Hui-Hua Yang ${ }^{1}$, Ge Li ${ }^{4}$, Xun-Hua Li ${ }^{1}$, Chao Wu', Qing-Ling Fu ${ }^{2^{*}}$ and Zhong Pei ${ }^{1^{*}}$

\begin{abstract}
Background: Machado-Joseph disease is the most common autosomal dominant hereditary ataxia worldwide without effective treatment. Mesenchymal stem cells (MSCs) could slow the disease progression, but side effects limited their clinical application. Besides, MSC-derived exosomes exerted similar efficacy and have many advantages over MSCs. The aim of this study was to examine the efficacy of MSC-derived exosomes in YACMJD84.2 mice.

Methods: Rotarod performance was evaluated every 2 weeks after a presymptomatic administration of intravenous MSC-derived exosomes twice in YACMJD84.2 mice. Loss of Purkinje cells, relative expression level of Bcl-2/Bax, cerebellar myelin loss, and neuroinflammation were assessed 8 weeks following treatment.

Results: MSC-derived exosomes were isolated and purified through anion exchange chromatography. Better coordination in rotarod performance was maintained for 6 weeks in YACMJD84.2 mice with exosomal treatment, compared with those without exosomal treatment. Neuropathological changes including loss of Purkinje cells, cerebellar myelin loss, and neuroinflammation were also attenuated 8 weeks after exosomal treatment. The higher relative ratio of $\mathrm{BCl}-2 / \mathrm{Bax}$ was consistent with the attenuation of loss of Purkinje cells.
\end{abstract}

Conclusions: MSC-derived exosomes could promote rotarod performance and attenuate neuropathology, including loss of Purkinje cells, cerebellar myelin loss, and neuroinflammation. Therefore, MSC-derived exosomes have a great potential in the treatment of Machado-Joseph disease.

Keywords: Machado-Joseph disease, Mesenchymal stem cell-derived exosomes, Motor function, Neuropathology

\footnotetext{
*Correspondence: fuqingl@mail.ysu.edu.cn; peizhong@mail.sysu.edu.cn

${ }^{+}$Hua-Jing You and Shu-Bin Fang contributed equally to this work.

${ }^{2}$ Otorhinolaryngology Hospital, The First Affiliated Hospital, Sun Yat-sen

University, No. 58 Zhongshan Road 2, Guangzhou 510080, Guangdong,

China

'Department of Neurology, The First Affiliated Hospital, Sun Yat-sen

University; Guangdong Provincial Key Laboratory of Diagnosis and Treatment

of Major Neurological Diseases, National Key Clinical Department and Key

Discipline of Neurology, No. 58 Zhongshan Road 2, Guangzhou 510080,

Guangdong, China

Full list of author information is available at the end of the article
}

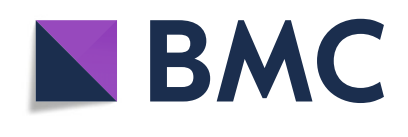

( ) The Author(s). 2020 Open Access This article is licensed under a Creative Commons Attribution 4.0 International License, which permits use, sharing, adaptation, distribution and reproduction in any medium or format, as long as you give appropriate credit to the original author(s) and the source, provide a link to the Creative Commons licence, and indicate if changes were made. The images or other third party material in this article are included in the article's Creative Commons licence, unless indicated otherwise in a credit line to the material. If material is not included in the article's Creative Commons licence and your intended use is not permitted by statutory regulation or exceeds the permitted use, you will need to obtain permission directly from the copyright holder. To view a copy of this licence, visit http://creativecommons.org/licenses/by/4.0/. The Creative Commons Public Domain Dedication waiver (http://creativecommons.org/publicdomain/zero/1.0/) applies to the data made available in this article, unless otherwise stated in a credit line to the data. 


\section{Background}

Machado-Joseph disease (MJD), also known as spinocerebellar ataxia type 3 (SCA3), is the most common autosomal dominant hereditary ataxia worldwide, reaching $40-70 \%$ in Japan, China, and Portugal [1, 2]. Clinical symptoms include progressive cerebellar ataxia, ophthalmoplegia, dysarthria, spasticity, and peripheral neuropathy. It is caused by CAG repeat expansion in the MJD1 gene, which encodes ATAXN3 protein. Mutant ATXN3 protein aggregates in neurons, forms nuclear inclusions, and disturbs the ubiquitin-proteasome pathway, leading to neurodegeneration, neuroinflammation, and brain atrophy especially in the cerebellar nuclei, brainstem, and basal ganglia [3, 4].

Given that there are currently no effective treatments for MJD, many attempts have been made to develop effective therapies to slow and stop this disease. Mesenchymal stem cells (MSCs) are multipotent stem cells that can differentiate into different cell types in the brain and release many potent factors. Since MSCs are easily obtained and expanded in vitro, MSC-based cell therapy has been extensively investigated in many neurological diseases, including MJD [5-9]. However, the clinical application of MSCs is hindered by side effects such as risks of oncogenicity and cellular embolism $[10,11]$. Recently, increasing evidence has suggested that MSCs exert their therapeutic effects largely through paracrine secretion, such as exosomes. Exosomes are small vesicles of $30-100 \mathrm{~nm}$ in diameter that contain many cytokines and microRNAs [12]. MSC-derived exosomes have many advantages over MSCs, including higher efficiency of passing through the blood-brain barrier, longer half-life period, lower immunogenicity, higher stability, and easier storage and transportation conditions [13]. Their effects have been proven to be comparable with MSCs in different models of neurological diseases [14, 15].

In the present study, we aim to investigate whether MSC-derived exosomes can slow down the disease progression in a transgenic mouse model of MJD. We evaluated rotarod performance every 2 weeks and examined the loss of Purkinje cells, myelin loss, and neuroinflammation after exosomal treatment. We found that exosomes could improve rotarod performance, as well as attenuate neuropathology including loss of Purkinje cells, demyelination, and neuroinflammation. The present study suggests a promising potential of MSC-derived exosomes in the treatment of MJD.

\section{Methods}

\section{Cell culture}

Human urine cell-derived induced pluripotent stem cells (U-iPSCs) were donated by the Guangzhou Institute of Biomedicine and Health, Chinese Academy of Science, Guangzhou, China [16]. Human MSCs were generated from U-iPSCs according to the methods of our previous study and were passaged and cryopreserved at P10 at s density of $2 \times 10^{6}$ per vial $[16,17]$. The characteristics of iPSC-MSCs were the specific fibroblastic morphology; positive for CD105, CD73, CD146, CD144, and CD44; and negative for CD3, CD14, CD19, and CD45 (supplementary Fig. 1S). One vial of MSCs was thawed and cultured in two $150-\mathrm{cm}^{2}$ cell culture plates and incubated with cell culture medium (CCM), as previously reported [16]. After 2-3 days, when the density of MSCs reached $\sim 80 \%$, the cells were further cultured in $25150-\mathrm{cm}^{2}$ cell culture plates and were incubated for 3-4 days. MSCs were then washed in phosphate-buffered saline (PBS) three times, and CCM was then replaced with chemically defined and protein-free (CDPF) medium as in our previous study [18], which contains $\mathrm{CD}-\mathrm{CHO}$ medium (catalog number 10743-029, Gibco, USA), HT supplement (catalog umber 11067-030, Gibco, USA), L-glutamine (catalog number 25030-081, Gibco, USA), D(+)-glucose (catalog number 50-99-7, Sigma, USA), nonessential amino acid (catalog number 11140-05, Gibco, USA), and Vitamin Solution (catalog number 11120-052, Gibco, USA). CDPF medium was replaced with fresh CDPF medium $6 \mathrm{~h}$ later, and MSCs were cultured for another 42 $\mathrm{h}$. The supernatants from 6 to $48 \mathrm{~h}$ were collected and used for subsequent isolation of MSC-derived exosomes.

\section{Exosome isolation}

MSC-derived exosomes were isolated and purified through anion exchange chromatography as in our previous study [18]. Briefly, the chromatographic column was packed with $4 \mathrm{~mL} \mathrm{Q}$-sepharose and then washed with $12 \mathrm{~mL}$ equilibration buffer $(50 \mathrm{mM}$ phosphate buffer, $100 \mathrm{mM} \mathrm{NaCl}$ ). Next, $150 \mathrm{~mL}$ supernatant containing MSC exosomes was loaded onto the column, which was washed with $40 \mathrm{~mL}$ wash buffer $(50 \mathrm{mM}$ phosphate buffer, $50 \mathrm{mM} \mathrm{NaCl}$ ) to remove protein impurities. Bound material was then eluted with $1 \mathrm{~mL}$ elution buffer (50 mM phosphate buffer, $500 \mathrm{mM} \mathrm{NaCl}$ ) for eight times. The concentrations of exosomes in these fractions were determined by Bradford Protein Assay, and the peak fractions were collected for dialysis. The final concentration of exosomes was determined by nanoparticle tracking analysis (Nanosight NS300; Malvern, UK). The MSC-derived exosomes were collected and stored at $80{ }^{\circ} \mathrm{C}$ for further study (Fig. 1a).

\section{Identification of MSC exosomes}

To positively identify exosomes, iPSC-MSC exosomes were denatured in SDS loading buffer for $5 \mathrm{~min}$ at $95^{\circ} \mathrm{C}$ and then $10 \mu \mathrm{g}$ of total protein was loaded and separated by SDS-PAGE on $10 \%$ gels. The proteins were transferred onto the PVDF membranes and stained with primary antibodies against CD63 (1:2000, catalog number 


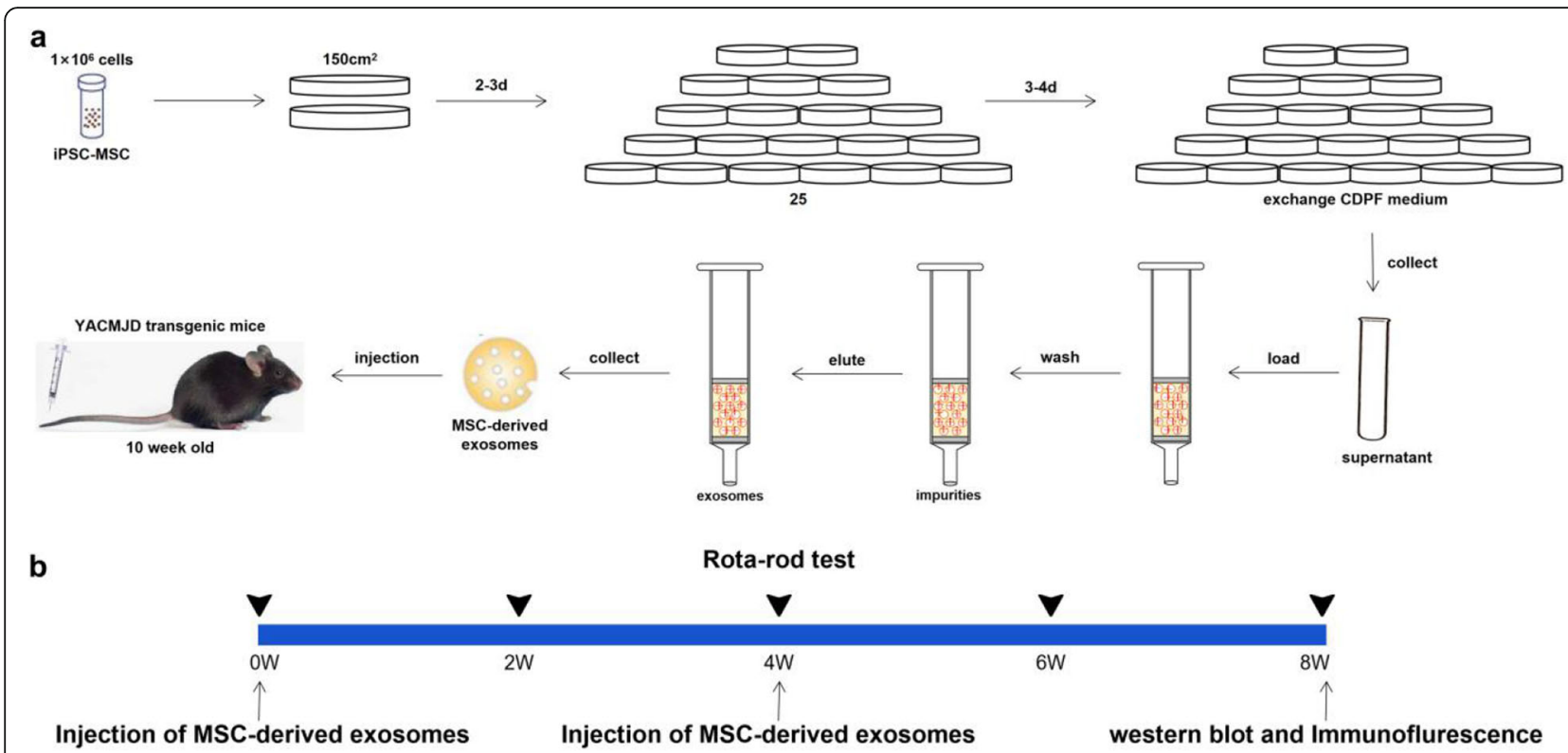

Fig. 1 Longitudinal trial design to extract MSC-derived exosomes and assess their effects. a Schematic presentation including the proliferation of iPSC-MSCs, collection of supernatant, anion chromatography, and tail vein injection of MSC-derived exosomes. $\mathbf{b}$ Timeline to analyze motor function, western blot, and immunofluorescence staining after injection of MSC-derived exosomes twice in YACMJD84.2 mice

Ab134045, Abcam, USA), Alix (1:5000, catalog number Ab186429, Abcam, USA), and TSG101 (1:1000, catalog number Ab125011, Abcam, USA) overnight at $4{ }^{\circ} \mathrm{C}$ after blocking with $4 \%$ skim milk. Then, the membranes were stained with goat anti-rabbit IgG HRP-conjugated antibody (1:2000, catalog number cw0103s, CWBIO) for $1 \mathrm{~h}$ and detected with Immobilon ${ }^{\mathrm{Tm}}$ Western Chemiluminescent HRP substrate (Millipore, Billerica, MA, USA).

\section{Animals}

All experimental procedures were in accordance with the guidelines of the Animal Research Committee of The First Affiliated Hospital, Sun Yat-sen University, Guangzhou, China, for the use and care of laboratory animals. YACMJD84.2 mice were generously provided by Professor Hong Jiang (Xiangya Hospital, Central South University, Changsha, China). The mice were generated in a C57BL/ 6 background strain and fed in a standard 12-h light/dark cycle with food and water ad libitum in the Laboratory Animals Monitoring Institute of Guangdong Province. Genotype identification was performed from DNA isolated from the tail tips using a Genomic DNA Mini Preparation Kit with Spin Column (Beyotime, China). Along with a pair of primers that amplified across CAG repeats in exon 10 of the human MJD1 gene (MJD52 5'-CCAGTGACTACTTTGATT CG-3'/MJD25 5'-TGGCCTTTCACATGGATGTGAA3'), Q84 hemizygosity was determined by quantitative real-time PCR using Premix Taq ${ }^{\mathrm{TM}}$ (Takara, Japan).

\section{Injection of MSC-derived exosomes}

Tail vein injection was performed on 10-week-old YACMJDQ84.2 mice. MSC-derived exosomes $(150 \mu \mathrm{L}$, approximately $1.5 \times 10^{10}$ per dose) were transplanted at 0 and 4 weeks. Motor function and neuropathology were compared among YACMJD84.2 mice injected with MSCderived exosomes (exosome-Tg, $n=8$ ), YACMJD84.2 mice without injection (Control-Tg, $n=8$ ), and wild-type mice without injection (Control-Wt, $n=8$ ) (Fig. 1b).

\section{Behavior test: rotarod test}

Passive movement and motor coordination were evaluated with a rotarod apparatus (Leica). Three groups of mice were placed on the rotarod at an accelerated speed (4-40 rpm, $5 \mathrm{~min}$ ), and the latency to fall was recorded. The mean latency to fall over three trials was calculated. The rotarod test was performed every 2 weeks at a similar time point.

\section{Tissue preparation}

After intraperitoneal injection of chloral hydrate, all mice were intracardiacally perfused with $50 \mathrm{~mL}$ of PBS ( $\mathrm{pH} 7.4$ ) at 18 weeks. One hemisphere was removed sagittally and fixed with cold $4 \%$ paraformaldehyde/PBS overnight at $4{ }^{\circ} \mathrm{C}$. The hemispheres were then dehydrated with $20 \%$ and $30 \%$ sucrose/PBS successively at $4{ }^{\circ} \mathrm{C}$ and embedded in the Tissue-Tek OCT compound (Sakura Finetek Japan, Tokyo, Japan). Finally, each cerebellum was sagittally or coronally sectioned with a 
frozen microtome (Leica) and stored at $-80^{\circ} \mathrm{C}$ until further use.

\section{Immunohistochemistry}

After rewarming, the sections were incubated in $0.3 \%$ Triton $^{\text {TM }}$ X-100 and Immunol Staining Blocking buffer (Beyotime, China) for $1 \mathrm{~h}$ at room temperature. The sections were then incubated overnight at $4{ }^{\circ} \mathrm{C}$ with the following primary antibodies: mouse anti-calbindin D28K (1:500, catalog number C9848, Sigma-Aldrich, USA), mouse anti-ATXN3 (1H9) (1:200, catalog number MAB5360, Millipore, USA), rabbit anti-glial fibrillary acidic protein (GFAP) (1:200, catalog number ab48050, Abcam, USA), and rat anti-myelin basic protein (MBP) (1:200, catalog number ab7349, Abcam, USA), followed by incubation with the following secondary antibodies: goat-anti mouse IgG $(\mathrm{H}+\mathrm{L}), \mathrm{F}(\mathrm{ab}) 2$ fragment (Alexa Fluor $^{\circ} 488$ conjugated) antibody (1:300, catalog number 4408S, Cell Signaling, USA), goat-anti mouse IgG $(\mathrm{H}+\mathrm{L})$, $\mathrm{F}\left(\mathrm{ab} \mathrm{b}^{\prime}\right) 2$ fragment (Alexa Fluor 555 conjugated) antibody (1:300, catalog number 4409S, Cell Signaling), goat-anti rabbit IgG $(\mathrm{H}+\mathrm{L}), \mathrm{F}(\mathrm{ab}) 2$ fragment (Alexa Fluor ${ }^{\circ} 488$ conjugated) antibody (1:300, catalog number $4412 \mathrm{~S}$, Cell Signaling), and goat-anti rat IgG $(\mathrm{H}+\mathrm{L}), \mathrm{F}\left(\mathrm{ab} \mathrm{b}^{\prime}\right) 2$ fragment (Alexa Fluor ${ }^{\circ} 488$ conjugated) antibody (1:300, catalog number 4416S, Cell Signaling) for $1 \mathrm{~h}$ at room temperature. Finally, DAPI was used to identify the nuclei. Immunofluorescence images were obtained with a confocal laser scanning microscope (Leica) or a twophoton confocal laser scanning microscope (Leica).

\section{Quantification of Purkinje cells}

Quantification of calbindin-positive Purkinje cells was performed by scanning three coronal sections in each group under the fluorescence microscope $(n=4)$. For each section, three fixed concave lobules were selected [19]. Linear density of calbindin-positive Purkinje cells along the cerebral cortex was determined as the number of Purkinje cells $/ \mathrm{mm}$ [20]. The average cell numbers and the relative ratio of calbindin-positive Purkinje cells compared with YACMJD84.2 mice without exosomal injection were calculated using ImageJ (National Institutes of Health, Bethesda, MD, USA).

\section{Quantification of fluorescence intensities}

Fluorescence intensities of MBP were analyzed by scanning three fixed cerebellar lobules in three coronal sections of each group [21]. The signal intensity of MBP in granular layers and white matter $/ 0.25 \mathrm{~mm}^{2}$ was calculated using ImageJ. Signal intensity in wild-type mice was used as the reference to calculate the relative ratio in the other two groups. The number of nuclear inclusions (1H9) and GFAP-positive fibers of the surrounding astrocytes $/ 0.25 \mathrm{~mm}^{2}$ in cerebellar nuclei was also evaluated similarly using Image).

\section{Western blot}

Protein lysates from the cerebellums were obtained using RIPA Lysis and Extraction Buffer (Thermo Scientific, Rockford, IL, USA) containing protease inhibitors (1:100, PMSF, Solarbio, China). After sonication and centrifugation at $12000 \mathrm{~g}$ for $15 \mathrm{~min}$ at $4{ }^{\circ} \mathrm{C}$, the supernatants were collected. The protein concentration was determined with the Pierce ${ }^{\mathrm{ms}}$ BCA Protein Assay Kit (Thermo Scientific) and adjusted to the same concentration. All samples were stored at $-80{ }^{\circ} \mathrm{C}$. Fifty micrograms of total proteins per sample was separated by 12.5\% SDS-PAGE gels (PAGE Gel Fast Preparation Kit, catalog number PG113, Epizyme, China) according to the manufacturer's protocol. Then, the proteins were transferred onto the PVDF membranes (Merck Millipore, Carrigtwohill, Ireland), followed by blocking with $5 \%$ skim milk for $1 \mathrm{~h}$ at room temperature. Rat antiMBP (1:2000, catalog number ab7349, Abcam, USA), rabbit Bcl-2 (1: 1000, catalog number 3498S, Cell Signaling, USA), rabbit Bax (1: 2000, catalog number 14796S, Cell Signaling, USA), and mouse anti-ATXN3 (1H9) (1: 1000, catalog number MAB5360, Millipore, USA) were incubated overnight at $4{ }^{\circ} \mathrm{C}$. The membranes were then incubated with goat anti-rat IgG $(\mathrm{H}+\mathrm{L}) \mathrm{HRP}$-linked antibody (1:1000, catalog number S0009, Affinity Biosciences, USA) for $1 \mathrm{~h}$ at $37^{\circ} \mathrm{C}$ and were detected by using Immobilon $^{\text {Tu }}$ Western Chemiluminescent HRP substrate (Millipore, Billerica, MA, USA).

\section{Statistical analysis}

All experimental data are presented as the mean \pm standard deviation/standard error of the mean for at least three independent experiments. The results were compared using one-way ANOVA. A $p \leq 0.05$ was considered significant statistically.

\section{Results}

\section{Characterization of MSC-derived exosomes}

The total concentration and volumes of MSC-derived exosomes used in the current study were approximately $1.0 \times 10^{11} / \mathrm{mL}$ and $200 \mu \mathrm{g} / \mathrm{mL}$, as determined by nanoparticle tracking analysis and Bradford protein assay, respectively. The diameter of MSC-derived exosomes was approximately $100 \mathrm{~nm}$ (Fig. 2a). Compared with MSCs, western blot analysis revealed that MSC-derived exosomes were positive for specific exosomal markers, including CD63, TSG101, and Alix (Fig. 2b). Additionally, transmission electron microscopy showed that they were bilipidlayered particles (Fig. 2c). Taken together, these results demonstrated that high-quality MSC-derived exosomes could be isolated using anion exchange chromatography. 

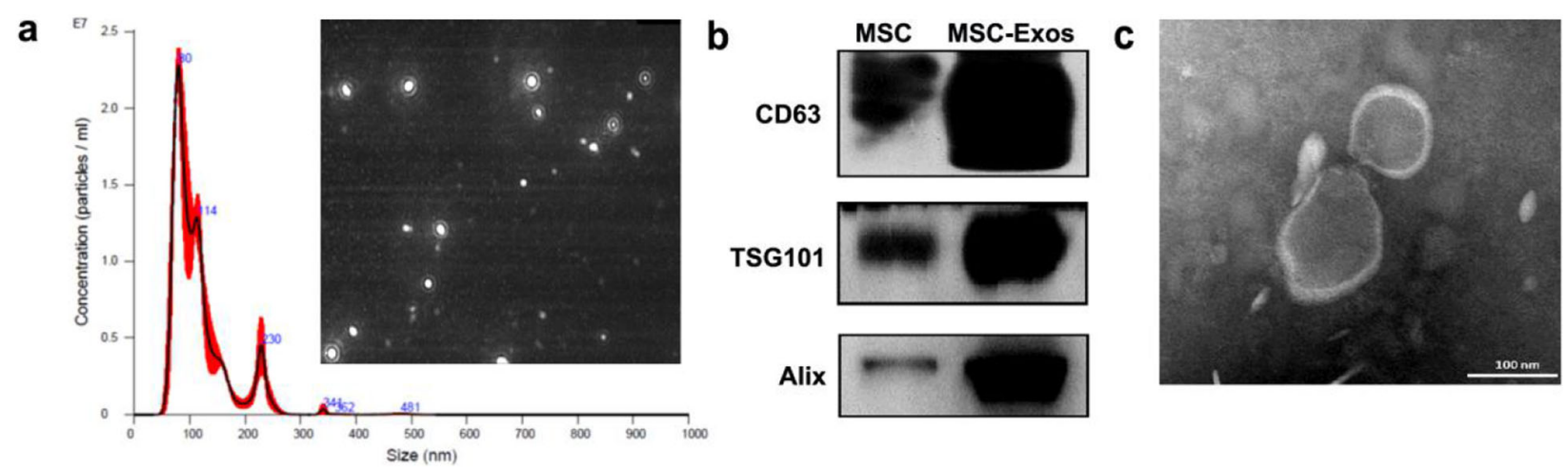

Fig. 2 Characterization of exosomes derived from iPSC-MSCS. a The NTA of iPSC-MSC exosomes. b Compared with MSCS, the iPSC-MSC exosomes were strongly positive for CD63, TSG101, and Alix as determined by western blot. c The iPSC-MSC exosomes were bilipid-layered particles with a diameter of about $100 \mathrm{~nm}$ as shown by TEM

\section{MSC-derived exosomes improved motor function}

To assess the effects of MSC-derived exosomes on motor function, exosomes were intravenously administrated into the tail vein of YACMJD84.2 mice. The rotarod test was used to evaluate motor function every 2 weeks until 8 weeks after treatment.

In the first 2 weeks, there was no significant difference in motor function among the normal controls (ControlWt), YACMJD84.2 mice with exosomal treatment (Exosome-Tg), or those without exosomal treatment (Control$\mathrm{Tg}$ ). Four weeks after treatment, rotarod performance deteriorated in YACMJD84.2 mice without exosomal treatment (Control-Tg $261.837 \pm 9.418 \mathrm{~s}$ ). In contrast, the rotarod performance of YACMJD84.2 mice with exosomal treatment remained similar to normal control (ExosomeTg $274.038 \pm 5.608 \mathrm{~s}$ versus Control-Wt $279.050 \pm 7.990$ s). Six weeks after treatment, the deterioration of rotarod performance was more severe in YACMJD84.2 mice without exosomal treatment, compared with YACMJD84.2 mice with exosomal treatment and also with normal controls (Control-Wt $282.962 \pm 7.656 \mathrm{~s}$ versus Exosome-Tg $279.675 \pm 6.169 \mathrm{~s}$ versus Control-Tg $262.262 \pm 9.597 \mathrm{~s}, p=$ 0.024). Eight weeks after treatment, rotarod performance deteriorated in YACMJD84.2 mice with exosomal treatment compared with normal controls (Exosome-Tg $263.487 \pm 11.363 \mathrm{~s}$ versus Control-Wt $283.875 \pm 11.487 \mathrm{~s}$, $p=0.014)$. However, the difference in rotarod performance still remained significant between YACMJD84.2 mice with and without exosomal treatment (Exosome-Tg $263.487 \pm 11.363 \mathrm{~s}$ versus Control-Tg $242.212 \pm 20.538 \mathrm{~s}$ ).

\section{MSC-derived exosomes attenuated apoptosis of Purkinje cells}

Apoptosis of Purkinje cells, which is an important type of cell degeneration in MJD, was evaluated 8 weeks after exosomal treatment. Purkinje cells are responsible for cerebellar function. Consistent with the observations in both MJD patients and animal models, YACMJD84.2 mice exhibited a remarkable loss of Purkinje cells [22]. MSC-derived exosomes significantly attenuated the loss of Purkinje cells. The number of calbindin-positive Purkinje cells was $1.52 \pm 0.20$-fold higher in YACMJD84.2 mice with exosomal treatment than in those without exosomal treatment (Exosome-Tg $15.20 \pm 1.73$ versus Control-Tg $10.00 \pm 1.89, p=0.022$, Fig. 3b, c). Although the number of Purkinje cells in YACMJD84.2 mice with exosomal treatment was slightly lower relative to normal controls, the difference was not significant (Control-Wt $16.20 \pm 2.61$ versus Control-Tg $15.20 \pm 1.73, p>0.05$ ).

To explore the possible mechanism of the effect on Purkinje cells, the relative expression level of Bcl-2 and Bax was analyzed. The expression level of anti-apoptotic gene $\mathrm{Bcl}-2$ in mice with exosomal treatment was higher than those without treatment, while the expression of pro-apoptotic gene Bax was just the opposite (Fig. 3d). Therefore, the relative ratio of $\mathrm{Bcl}-2 / \mathrm{Bax}$ in mice with exosomal treatment was significantly higher (Exosome$\mathrm{Tg} 0.75 \pm 0.054$ versus Control-Tg $0.56 \pm 0.054$ versus Control-Wt $0.47 \pm 0.031 \mathrm{~s}, p=0.0073$, Fig. 3e).

\section{MSC-derived exosomes attenuated myelin loss}

The effect of MSC-derived exosomes on myelin loss was also evaluated 8 weeks after treatment. Myelin loss due to axonal inclusions and impaired axonal transportation are evident in MJD patients and animals [23, 24]. Myelin basic protein (MBP) is the main component of the myelin sheath, which surrounds axons in a molecular layer within the cerebellar white matter. The morphological changes and expression of MBP were assessed by immunofluorescence and western blot, respectively. Quantitative analysis of fluorescence intensity was performed following a previous report [25]. The relative intensity of MBP signals was $1.494 \pm 0.211$ and $0.999 \pm 0.217$ in YACMJD84.2 mice with and without exosomal treatment, respectively (Fig. 4a, 

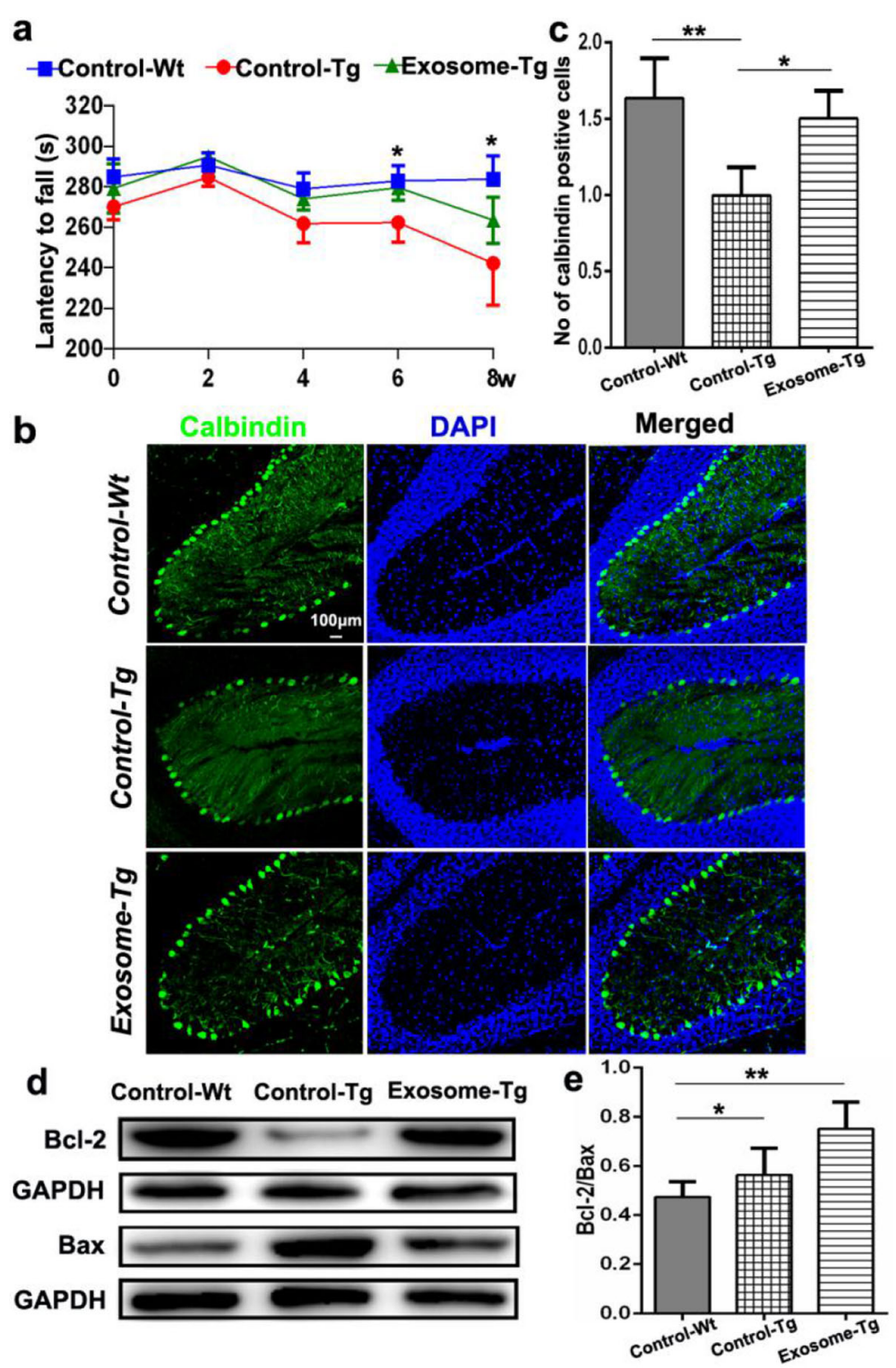

Fig. 3 Injection of MSC-derived exosomes significantly improved motor function and rescued apoptosis of Purkinje cells. a Rotarod test was performed at an accelerated velocity in YACMJD84.2 mice with exosomal treatment (Exosome-Tg, $n=8$ ), YACMJD84.2 (Control-Tg, $n=8)$, and wild-type littermates (Control-Wt, $n=8$ ). Data was presented as mean \pm SEM. b, c Quantitative and histological analysis of calbindin-positive cells in the sagittal cerebellar sections 8 weeks after injection $(n=4)$. The pictures were captured with a confocal laser scanning microscope at $\times 20$ magnification. The scale bar was $100 \mu \mathrm{m}$. d, e Quantitative and western blot analysis of relative expression of Bcl-2/Bax protein ( $n=4)$. One-way ANOVA analysis; ${ }^{*} p \leq 0.05,{ }^{* *} p \leq 0.01$

b). The results were further confirmed by western blot. The expression of MBP was also significantly higher in YACMJD84.2 mice with exosomal treatment than in those without exosomal treatment (Fig. 4c; Exosome-Tg $0.976 \pm$ 0.060 versus Control-Tg $0.737 \pm 0.199, p=0.0185$ ). Besides, signal intensity and expression of MBP in YACMJD84.2 mice with exosomal treatment were both slightly lower than those in normal controls, although these levels were not significant.
MSC-derived exosomes attenuated neuroinflammation without reducing nuclear inclusions

Inflammation and nuclear inclusions (NIs) associated with mutant ATXN3 in the brainstem are key components of MJD neuropathology [26, 27]. The effects of exosomal treatment on astrocytes and NIs were explored by immunofluorescence staining. The number of activated astrocytes was lower in YACMJD84.2 mice with exosomal treatment than in those without exosomal 


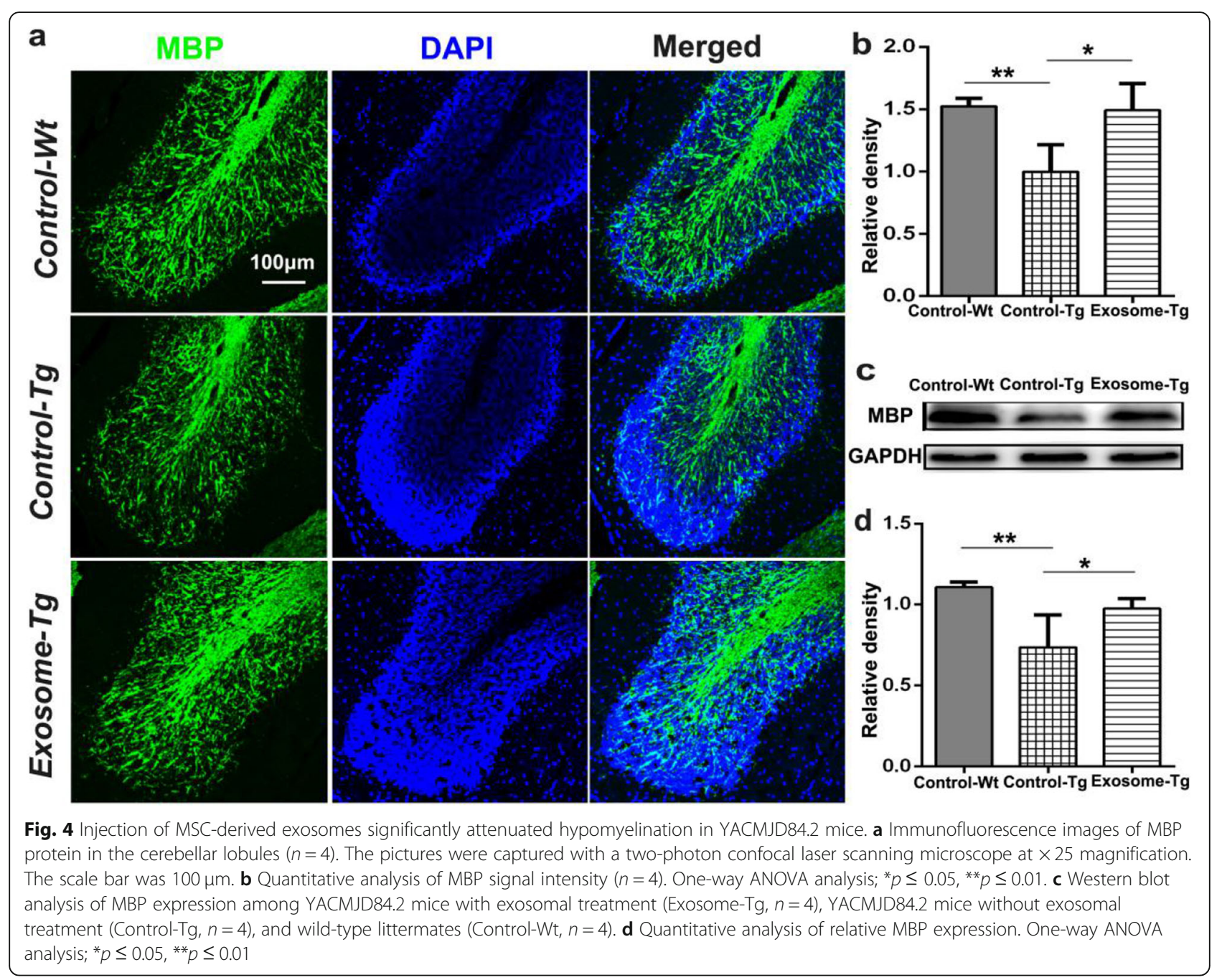

treatment, especially in the pons (Fig. 5a, b; Exosome-Tg $11.51 \pm 3.281$ versus Control-Tg $18.94 \pm 2.983$ versus Control-Wt $5.978 \pm 2.320, p=0.005)$. However, there was no significant difference in the number of NIs between these two groups (Fig. 5c). We further examined the expression of ATXN3 protein in the cerebellum by western blot. There was no difference in the expression levels of mutant ATXN3 between YACMJD84.2 mice with and without exosomal treatment (Fig. 5d, e).

\section{Discussion}

MJD is a fatal polyglutamine neurodegenerative disease with no cure. In the present study, we evaluated the therapeutic potential of MSC-derived exosomes in a mouse model of MJD. Our result showed that MSCderived exosomes can be isolated easily using anion exchange chromatography. In addition, MSC-derived exosomes can alleviate motor deficits and neuropathological changes in YACMJD84.2 mice. Thus, MSC-derived exosomes may have a great potential to be an effective therapy for MJD.

One of the more significant obstacles for exosomebased therapy is the difficulty with which to isolate sufficient numbers of exosomes. Ultracentrifugation is the most common method used to isolate exosomes; however, isolation of exosomes via ultracentrifugation is time-consuming [13]. Other disadvantages include possible sample contamination and damage to the architecture and components of exosomes. Compared with ultracentrifugation, the yield rate of exosomes isolated by anion exchange chromatography was nearly 2 times higher with only $20 \%$ of the time costing in our previous study [18]. Consistently, we achieved a high yield of good-quality exosomes from MSCs using anion exchange chromatography. The final concentration and volume, which were as high as $1.0 \times 10^{11} / \mathrm{mL}$ and $200 \mu \mathrm{g} / \mathrm{mL}$, respectively, may have a great potential to satisfy future requirements in clinical application. 


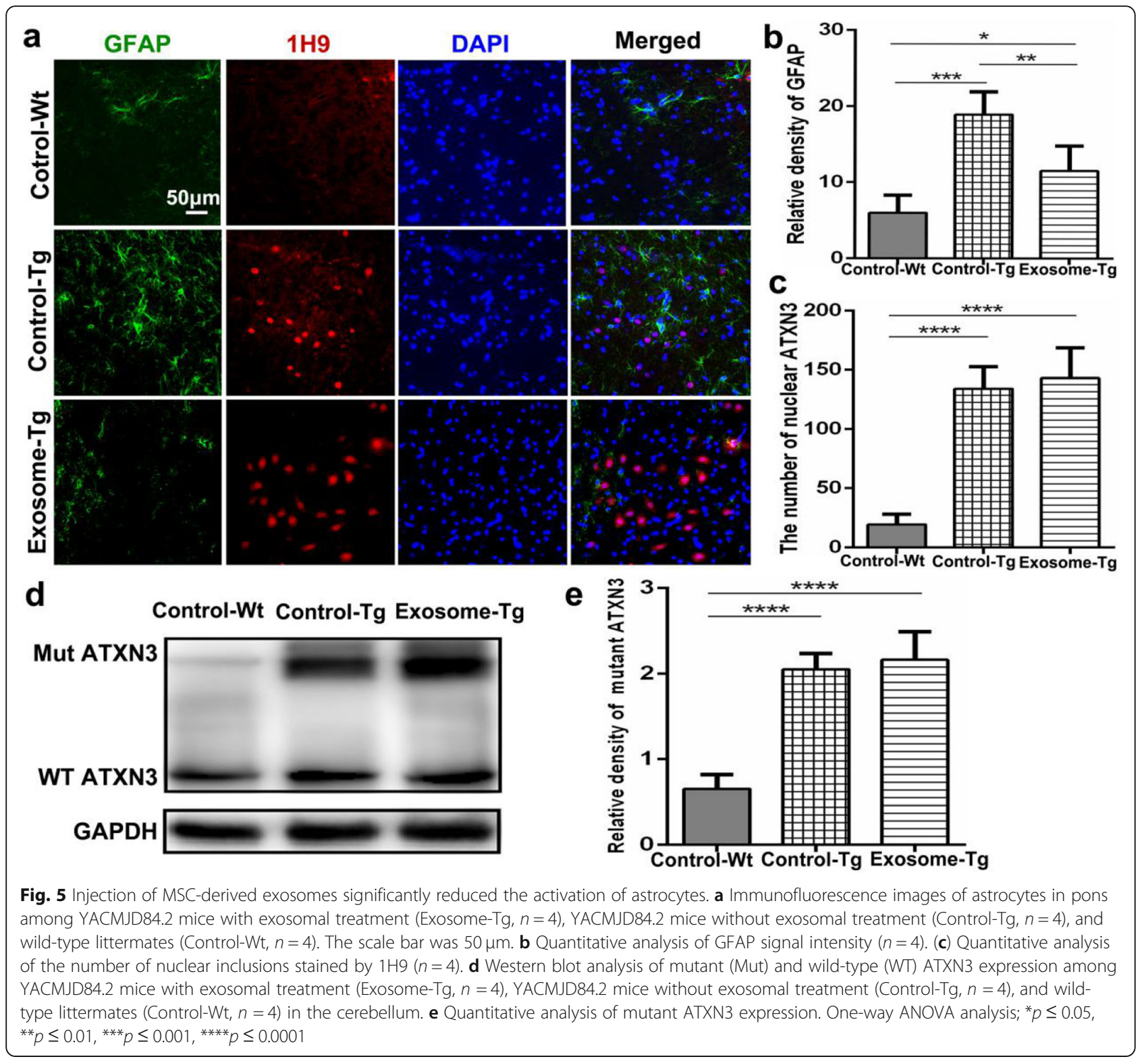

The timing of intravenous administration is important and may influence the efficacy of exosomes. Similar to other polyglutamate diseases, the pathological alterations occur before disease onset in MJD patients and animal models [26, 28]. Therefore, pre-symptomatic treatment may be necessary. Indeed, MSC treatment failed to attenuate motor dysfunction and neuropathology when it was given after disease onset [29]. In the present study, a pretreatment paradigm was adopted to test the possible efficacy of exosomes in YACMJD84.2 mice. YACMJD84.2 mice carry the yeast artificial chromosome (YAC) constructed with $(\mathrm{CAG})_{84}$ and all necessary regulatory elements [30]. These mice are widely used in preclinical trials because they recapitulate typical motor phenotypes, as well as neuropathological features associated with MJD.
In the present study, YACMJD84.2 mice developed motor deficits as evidenced by the decline in rotarod performance at the age of 14 weeks, which was consistent with a previous study [28]. Intravenous administration of exosomes significantly prolonged normal motor function for 4 weeks and attenuated motor deficits for an additional 2 weeks, when compared with YACMJD84.2 mice without exosomal treatment. Previously, mice receiving intravenous MSCs exhibited better motor function starting from 7 to 8 weeks after treatment. The beneficial effects of intravenous MSCs were also shown to last for 4-6 weeks [8, $31,32]$. By comparison, the protective effects of between exosomes and MSCs appear to be similar. Moreover, exosomes exerted effects starting from 4 weeks following treatment, which was 3 weeks faster than MSCs. These 
results suggest that exosomes may serve as a cell-free therapy to replace MSCs in the treatment of MJD. However, it should be noted that the protective effects decreased 8 weeks after treatment, although exosomes have a relatively long half-life, and suggests that a repetitive administration paradigm may be required to achieve a sustained alleviation of motor dysfunction [33].

Purkinje cells in the cerebellar cortex are key for motor coordination and send impulses that cooperate with deep cerebellar nuclei (DCN) and molecular effectors to regulate cerebellar neuronal circuits [34, 35]. Mutant ATXN3 expression induces degeneration of Purkinje cells, which in turn results in dyscoordination of MJD patients and animals [36]. Apoptosis is a major type of cell death in MJD. It is generally believed that the balance between proapoptotic Bax protein and anti-apoptotic Bcl-2 protein determine the fate of cells. Once activated, Bax oligomerizes at the mitochondrial outer membrane, induces permeability transition, and initiates apoptotic process. Positive immunoreactivity for Bax was found in the Purkinje cells of three MJD patients, indicating its possible pro-apoptotic mechanism in MJD [37]. In contrast, Bcl-2 blocks mitochondrial permeability transition and inhibits apoptotic process. In the present study, there was a noticeable loss of Purkinje cells and upregulated expression of Bax in the cerebellum of YACMJD84.2 mice. However, consistent with a previous study, exosomal treatment induced an increase in the relative expression of $\mathrm{Bcl}-2 / \mathrm{Bax}$ along with a decrease in cell loss, suggesting that exosome-induced high expression of $\mathrm{Bcl}-2 / \mathrm{Bax}$ may be responsible for the reduction of the Purkinje cell loss [38, 39].

Cerebellar afferent and efferent axons are responsible for the complicated connection between Purkinje cells, cerebellar nuclei, and the pons [40]. In the central nervous system (CNS), mutant ATAXN3 leads to hypomyelination, impairs axonal transportation, and induces death of interconnected neurons [22, 23, 30, 41]. MBP is the second most abundant protein and the only structural protein essential for myelination in the CNS [42]. MSCderived exosomes have been shown to promote proliferation, differentiation, and migration of oligodendrocytes, which in turn rescued damaged axons and improved recovery of neurological function in different models of neurological diseases [43, 44]. Consistently, exosomal treatment significantly upregulated MBP signal intensity and expression in the cerebellum of YACMJD84.2 mice compared with those without treatment. Thus, exosomes may improve motor function, at least partially through rescuing myelin loss.

Gliosis is a prominent neuropathological feature in MJD brains [45]. Activated astrocytes, a key player of neuroinflammation, have been linked to neurodegeneration in MJD. Consistently, activated astrocytes were evident in YACMJD84.2 mice, while activation of astrocytes was significantly reduced in those with exosomal treatment, indicating the anti-inflammatory function of exosomes $[46,47]$. Interestingly, mutant ATXN3 remained unchanged between mice with exosomes and those without exosomes. This observation is consistent with a previous report that MSC did not directly inhibit ATXN3 in a mouse model of MJD [9]. Thus, the protective mechanism by exosomes may not involve the inhibition of mutant ATXN3 protein. Future study is needed to reveal the antiinflammatory mechanism of MSC-derived exosomes.

There are some limitations in our study. First of all, we did not examine the spinal cord of mice with MSCderived exosomes. Given that the spinal cord can be affected in MJD, a future study on the efficacy of MSCderived exosomes in the spinal cord will provide significant insights into the functions of exosomes in the treatment of MJD. In addition, other markers of Purkinje cells such as Zebrin II and cerebellin can be added in the future study to provide a better assessment of Purkinje cells. Moreover, therapeutic mechanisms are less explored. Thoughtful comprehensive mechanistic studies will be required to understand the therapeutic mechanisms of MSC-derived exosomes in MJD.

\section{Conclusion}

We demonstrate that MSC-derived exosomes can improve motor function, reduce the loss of Purkinje cells, rescue the loss of myelin, and attenuate neuroinflammation. Collectively, MSC-derived exosomes are a promising therapeutic approach for MJD patients.

\section{Supplementary information}

Supplementary information accompanies this paper at https://doi.org/10. 1186/s13287-020-01727-2.

Additional file 1: Figure S1. Characterization of human induced pluripotent stem cell-derived mesenchymal stem cells. (a) Morphology of iPSC-MSCs (original magnification, 100x). (b) The MSC surface markers in iPSC-MSCs by flow cytometry. Cells were harvested at passage 9 .

\section{Abbreviations}

MJD: Machado-Joseph disease; SCA3: Spinocerebellar ataxia type 3; iPSCs: Induced pluripotent stem cells; CCM: Cell culture medium; PBS: Phosphate-buffered saline; CDPF: Chemically defined and protein-free; MBP: Myelin basic protein; NIs: Nuclear inclusions; DCN: Deep cerebellar nucleus; YAC: Yeast artificial chromosome; CNS: Central nervous system

\section{Acknowledgements}

The authors wish to thank Professor Hong Jiang and his team members from Xiangya Hospital, Central South University, Changsha, China, for providing YACMJD84.2 mice and methods of genotype identification. We also thank the International Science Editing (http://www.internationalscienceediting.com) for editing this manuscript.

\section{Authors' contributions}

H-JY, S-BF, T-TW, H-YZ, Y-KF, and X-JL performed the experiments. H-JY and S-BF drafted the manuscript. Q-LF and ZP designed the research, revised the manuscript, and approved the final version of the manuscript. All authors read and approved the manuscript. 


\section{Funding}

The study was supported by the grants from the National Natural Science Foundation of China (No. 81873751, 81671102); the National Key Research and Development Program of China, Stem Cell and Translational Research (No. 2017YFA0105104); Guangdong Provincial Science and Technology Plan Project (No. 2016B030230002, 2017A040406007); Guangdong Provincial Key Laboratory for Diagnosis and Treatment of Major Neurological Diseases (2017B030314103); the Southern China International Cooperation Base for Early Intervention and Functional Rehabilitation of Neurological Diseases (2015B050501003); Guangdong Provincial Engineering Center for Major Neurological Disease Treatment; and Guangdong Provincial Translational Medicine Innovation Platform for Diagnosis and Treatment of Major Neurological Disease; Guangdong Provincial Clinical Research Center for Neurological Diseases.

\section{Availability of data and materials}

The datasets generated and/or analyzed during the study are available from the corresponding authors on reasonable request.

\section{Ethics approval and consent to participate}

This study was approved by the Ethics Committee of The First Affiliated Hospital, Sun Yat-sen University, Guangzhou, China. All experimental procedures were in accordance with the guidelines of the Animal Research Committee of The First Affiliated Hospital, Sun Yat-sen University, Guangzhou, China, for the use and care of laboratory animals.

\section{Consent for publication}

Not applicable.

\section{Competing interests}

The authors declare that they have no competing interests.

\section{Author details}

${ }^{1}$ Department of Neurology, The First Affiliated Hospital, Sun Yat-sen University; Guangdong Provincial Key Laboratory of Diagnosis and Treatment of Major Neurological Diseases, National Key Clinical Department and Key Discipline of Neurology, No. 58 Zhongshan Road 2, Guangzhou 510080, Guangdong, China. ${ }^{2}$ Otorhinolaryngology Hospital, The First Affiliated Hospital, Sun Yat-sen University, No. 58 Zhongshan Road 2, Guangzhou 510080, Guangdong, China. ${ }^{3}$ Department of Neurology, The Fifth Affiliated Hospital, Sun Yat-sen University, No. 52 Meihua Road East, Zhuhai 519100, Guangdong, China. ${ }^{4}$ Guangdong Provincial Key Laboratory of Laboratory Animals, Guangdong Laboratory Animals Monitoring Institute, No. 11 Fengxin Road, Guangzhou 510663, Guangdong, China.

\section{Received: 13 October 2019 Revised: 19 April 2020} Accepted: 12 May 2020 Published online: 08 June 2020

\section{References}

1. Martins S, Calafell F, Gaspar C, Wong VC, Silveira I, Nicholson GA, Brunt ER, Tranebjaerg L, Stevanin G, Hsieh M, et al. Asian origin for the worldwidespread mutational event in Machado-Joseph disease. Arch Neurol. 2007;64: 1502-8.

2. Paulson H. Machado-Joseph disease/spinocerebellar ataxia type 3. Handb Clin Neurol. 2012;103:437-49.

3. Costa Mdo C, Luna-Cancalon K, Fischer S, Ashraf NS, Ouyang M, Dharia RM, Martin-Fishman L, Yang Y, Shakkottai VG, Davidson BL, et al. Toward RNAi therapy for the polyglutamine disease Machado-Joseph disease. Mol Ther. 2013;21:1898-908.

4. Costa Mdo C, Paulson HL. Toward understanding Machado-Joseph disease. Prog Neurobiol. 2012;97:239-57.

5. Chang YK, Chen MH, Chiang YH, Chen YF, Ma WH, Tseng CY, Soong BW, Ho $\mathrm{JH}$, Lee OK. Mesenchymal stem cell transplantation ameliorates motor function deterioration of spinocerebellar ataxia by rescuing cerebellar Purkinje cells. J Biomed Sci. 2011;18:54.

6. Rodríguez-Lebrón E, Costa Mdo C, Luna-Cancalon K, Peron TM, Fischer S, Boudreau RL, Davidson BL, Paulson HL. Silencing mutant ATXN3 expression resolves molecular phenotypes in SCA3 transgenic mice. Mol Ther. 2013;21: 1909-18.

7. Liu J, Yu D, Aiba Y, Pendergraff H, Swayze EE, Lima WF, Hu J, Prakash TP, Corey DR. ss-siRNAs allele selectively inhibit ataxin-3 expression: multiple mechanisms for an alternative gene silencing strategy. Nucleic Acids Res. 2013;41:9570-83.

8. Matsuura S, Shuvaev AN, lizuka A, Nakamura K, Hirai H. Mesenchymal stem cells ameliorate cerebellar pathology in a mouse model of spinocerebellar ataxia type 1. Cerebellum. 2014;13:323-30.

9. Oliveira Miranda C, Marcelo A, Silva TP, Barata J, Vasconcelos-Ferreira A, Pereira D, Nobrega C, Duarte S, Barros I, Alves J, et al. Repeated mesenchymal stromal cell treatment sustainably alleviates Machado-Joseph disease. Mol Ther. 2018;26:2131-51.

10. Katsha AM, Ohkouchi S, Xin H, Kanehira M, Sun R, Nukiwa T, Saijo Y. Paracrine factors of multipotent stromal cells ameliorate lung injury in an elastase-induced emphysema model. Mol Ther. 2011;19:196-203.

11. Tran C, Damaser MS. Stem cells as drug delivery methods: application of stem cell secretome for regeneration. Adv Drug Deliv Rev. 2015;82-83:1-11.

12. Ophelders DR, Wolfs TG, Jellema RK, Zwanenburg A, Andriessen P, Delhaas $T$, Ludwig AK, Radtke S, Peters V, Janssen L, et al. Mesenchymal stromal cellderived extracellular vesicles protect the fetal brain after hypoxia-ischemia. Stem Cells Transl Med. 2016;5:754-63.

13. Yu B, Zhang X, Li X. Exosomes derived from mesenchymal stem cells. Int J Mol Sci. 2014;15:4142-57.

14. Yu B, Kim HW, Gong M, Wang J, Millard RW, Wang Y, Ashraf M, Xu M. Exosomes secreted from GATA-4 overexpressing mesenchymal stem cells serve as a reservoir of anti-apoptotic microRNAs for cardioprotection. Int J Cardiol. 2015;182:349-60.

15. Drommelschmidt K, Serdar M, Bendix I, Herz J, Bertling F, Prager S, Keller M, Ludwig AK, Duhan V, Radtke $S$, et al. Mesenchymal stem cell-derived extracellular vesicles ameliorate inflammation-induced preterm brain injury. Brain Behav Immun. 2017;60:220-32.

16. Gao WX, Sun YQ, Shi J, Li CL, Fang SB, Wang D, Deng XQ, Wen W, Fu QL. Effects of mesenchymal stem cells from human induced pluripotent stem cells on differentiation, maturation, and function of dendritic cells. Stem Cell Res Ther. 2017:8:48.

17. Yao $Y$, Fan $X L$, Jiang D, Zhang Y, Li X, Xu ZB, Fang SB, Chiu S, Tse HF, Lian $Q$, et al. Connexin 43-mediated mitochondrial transfer of iPSC-MSCs alleviates asthma inflammation. Stem Cell Reports. 2018;11:1120-35.

18. Fang SB, Zhang HY, Wang C, He BX, Liu XQ, Meng XC, Peng YQ, Xu ZB, Fan $X L, W u Z J$, et al. Small extracellular vesicles derived from human mesenchymal stromal cells prevent group 2 innate lymphoid cell-dominant allergic airway inflammation through delivery of miR-146a-5p. J Extracell Vesicles. 2020;9:1.

19. Mendonca LS, Nobrega C, Hirai H, Kaspar BK, Pereira de Almeida L. Transplantation of cerebellar neural stem cells improves motor coordination and neuropathology in Machado-Joseph disease mice. Brain. 2015;138:320-35.

20. Roux S, Bailly Y, Bossu JL. Regional and sex-dependent alterations in Purkinje cell density in the valproate mouse model of autism. Neuroreport. 2019;30:82-8.

21. Torashima T, Koyama C, lizuka A, Mitsumura K, Takayama K, Yanagi S, Oue M, Yamaguchi $H$, Hirai $H$. Lentivector-mediated rescue from cerebellar ataxia in a mouse model of spinocerebellar ataxia. EMBO Rep. 2008;9:393-9.

22. Scherzed W, Brunt ER, Heinsen $H$, de Vos RA, Seidel K, Burk K, Schols L, Auburger G, Del Turco D, Deller T, et al. Pathoanatomy of cerebellar degeneration in spinocerebellar ataxia type 2 (SCA2) and type 3 (SCA3). Cerebellum. 2012;11:749-60.

23. Seidel K, den Dunnen WF, Schultz C, Paulson H, Frank S, de Vos RA, Brunt ER, Deller T, Kampinga HH, Rüb U. Axonal inclusions in spinocerebellar ataxia type 3. Acta Neuropathol. 2010;120:449-60.

24. Suga N, Katsuno M, Koike H, Banno H, Suzuki K, Hashizume A, Mano T, lijima $M$, Kawagashira $Y$, Hirayama M, et al. Schwann cell involvement in the peripheral neuropathy of spinocerebellar ataxia type 3. Neuropathol Appl Neurobiol. 2014;40:628-39.

25. Kim JH, Yu JE, Chang BJ, Nahm SS. Neonatal influenza virus infection affects myelination in influenza-recovered mouse brain. J Vet Sci. 2018;19:750-8.

26. Evert BO, Vogt IR, Kindermann C, Ozimek L, de Vos RA, Brunt ER, Schmitt I, Klockgether T, Wüllner U. Inflammatory genes are upregulated in expanded ataxin-3-expressing cell lines and spinocerebellar ataxia type 3 brains. J Neurosci. 2002;21:5389-96.

27. Moore LR, Rajpal G, Dillingham IT, Qutob M, Blumenstein KG, Gattis D, Hung $\mathrm{G}$, Kordasiewicz HB, Paulson HL, McLoughlin HS. Evaluation of antisense oligonucleotides targeting ATXN3 in SCA3 mouse models. Mol Ther Nucleic Acids. 2017;7:200-10.

28. Hsieh J, Liu JW, Harn HJ, Hsueh KW, Rajamani K, Deng YC, Chia CM, Shyu WC, Lin SZ, Chiou TW. Human olfactory ensheathing cell transplantation 
improves motor function in a mouse model of type 3 spinocerebellar ataxia. Cell Transplant. 2017;26:1611-21.

29. Rossignol J, Fink K, Davis K, Clerc S, Crane A, Matchynski J, Lowrance S, Bombard M, Dekorver N, Lescaudron L, et al. Transplants of adult mesenchymal and neural stem cells provide neuroprotection and behavioral sparing in a transgenic rat model of Huntington's disease. Stem Cells. 2014;32:500-9.

30. Cemal CK, Carroll CJ, Lawrence L, Lowrie MB, Ruddle P, Al-Mahdawi S, King RH, Pook MA, Huxley C, Chamberlain S. YAC transgenic mice carrying pathological alleles of the MJD1 locus exhibit a mild and slowly progressive cerebellar deficit. Hum Mol Genet. 2002;11:1075-94.

31. Jones J, Jaramillo-Merchan J, Bueno C, Pastor D, Viso-Leon M, Martinez S. Mesenchymal stem cells rescue Purkinje cells and improve motor functions in a mouse model of cerebellar ataxia. Neurobiol Dis. 2010;40:415-23.

32. Zhang MJ, Sun JJ, Qian L, Liu Z, Zhang Z, Cao W, Li W, Xu Y. Human umbilical mesenchymal stem cells enhance the expression of neurotrophic factors and protect ataxic mice. Brain Res. 2011;1402:122-31.

33. Sarko DK, McKinney CE. Exosomes: origins and therapeutic potential for neurodegenerative disease. Front Neurosci. 2017;11:82.

34. Buffo A, Rossi F. Origin, lineage and function of cerebellar glia. Prog Neurobiol. 2013;109:42-63.

35. White JJ, Sillitoe RV. Development of the cerebellum: from gene expression patterns to circuit maps. Wiley Interdiscip Rev Dev Biol. 2013;2:149-64.

36. Shakkottai VG, Do Carmo Costa M, Dell'Orco JM, Sankaranarayanan A, Wulff $\mathrm{H}$, Paulson HL. Early changes in cerebellar physiology accompany motor dysfunction in the polyglutamine disease spinocerebellar ataxia type 3. J Neurosci. 2011;31:13002-14.

37. Kumada S, Hayashi M, Mizuguchi M, Nakano I, Morimatsu Y, Oda M. Cerebellar degeneration in hereditary dentatorubral-pallidoluysian atrophy and Machado-Joseph disease. Acta Neuropathol. 2000;99:48-54.

38. Zhao Y, Sun X, Cao W, Ma J, Sun L, Qian H, Zhu W, Xu W. Exosomes derived from human umbilical cord mesenchymal stem cells relieve acute myocardial ischemic injury. Stem Cells Int. 2015;2015:761643.

39. Chen HC, Kanai M, Inoue-Yamauchi A, Tu HC, Huang Y, Ren D, Kim H, Takeda S, Reyna DE, Chan PM, et al. An interconnected hierarchical model of cell death regulation by the BCL-2 family. Nat Cell Biol. 2015;17:1270-81.

40. Fujita $\mathrm{H}$, Sugihara I. Branching patterns of olivocerebellar axons in relation to the compartmental organization of the cerebellum. Front Neural Circuits. 2013;7:3.

41. Bezerra EML, Pedroso JL, Pinheiro DS, Braga-Neto P, Barsottini POG, Braga $\mathrm{NI}$, Manzano GM. Pattern of peripheral nerve involvement in MachadoJoseph disease: neuronopathy or distal axonopathy? A clinical and neurophysiological evaluation. Eur Neurol. 2013;69:129-33.

42. Boggs JM. Myelin basic protein: a multifunctional protein. Cell Mol Life Sci. 2006;63:1945-61.

43. Otero-Ortega L, Laso-Garcia F, Gomez-de Frutos MD, Rodriguez-Frutos B, Pascual-Guerra J, Fuentes B, Diez-Tejedor E, Gutierrez-Fernandez M. White matter repair after extracellular vesicles administration in an experimental animal model of subcortical stroke. Sci Rep. 2017;7:44433.

44. Han Y, Seyfried D, Meng Y, Yang D, Schultz L, Chopp M, Seyfried D. Multipotent mesenchymal stromal cell-derived exosomes improve functional recovery after experimental intracerebral hemorrhage in the rat. J Neurosurg. 2018;131:290-300

45. Rüb U, Schöls L, Paulson H, Auburger G, Kermer P, Jen JC, Seidel K, Korf HW, Deller T. Clinical features, neurogenetics and neuropathology of the polyglutamine spinocerebellar ataxias type 1, 2, 3,6 and 7. Prog Neurobiol. 2013;104:38-66.

46. Long Q, Upadhya D, Hattiangady B, Kim DK, An SY, Shuai B, Prockop DJ, Shetty AK. Intranasal MSC-derived A1-exosomes ease inflammation, and prevent abnormal neurogenesis and memory dysfunction after status epilepticus. Proc Natl Acad Sci U S A. 2017;114:E3536-45.

47. Thomi G, Surbek D, Haesler V, Joerger-Messerli M, Schoeberlein A. Exosomes derived from umbilical cord mesenchymal stem cells reduce microgliamediated neuroinflammation in perinatal brain injury. Stem Cell Res Ther. 2019:10:105

\section{Publisher's Note}

Springer Nature remains neutral with regard to jurisdictional claims in published maps and institutional affiliations.

Ready to submit your research? Choose BMC and benefit from:

- fast, convenient online submission

- thorough peer review by experienced researchers in your field

- rapid publication on acceptance

- support for research data, including large and complex data types

- gold Open Access which fosters wider collaboration and increased citations

- maximum visibility for your research: over $100 \mathrm{M}$ website views per year

At BMC, research is always in progress.

Learn more biomedcentral.com/submissions 